\title{
Is Caries in the Early Mixed Dentition Associated with Caries Development during Orthodontic Treatment?
}

\author{
Valbona Soumas ${ }^{1}$, Stavros Kiliaridis ${ }^{1}$, Christine B. Staudt ${ }^{2}$ \\ ${ }^{1}$ Department of Orthodontics, School of Dentistry, University of Geneva, Geneva, Switzerland \\ ${ }^{2}$ Clinic of Orthodontics and Pediatric Dentistry, Center of Dental Medicine, University of Zurich, Zurich, \\ Switzerland \\ Email:valbona@soumas.name
}

Received 25 August 2015; accepted 3 November 2015; published 6 November 2015

Copyright (C) 2015 by authors and Scientific Research Publishing Inc.

This work is licensed under the Creative Commons Attribution International License (CC BY). http://creativecommons.org/licenses/by/4.0/

(c) (7) Open Access

\begin{abstract}
Objective: To investigate if caries in the pre-treatment early mixed dentition is associated with caries development in the permanent dentition during orthodontic treatment. Material and Methods: We included 41 consecutive patients ( 29 girls, 12 boys) with a pre-treatment documentation in the mixed (mean age 9.4 years) and a post treatment documentation in the permanent dentition (age 14.9 years) (two-phased treatment time $4.5( \pm 1.6)$ years). The DMFT/S indices were calculated. High-risk and low-risk groups were defined according to dmfs + DMFS score before treatment. Results: Initial dmft/s + DMFT/S (SD) was 5.15 (3.60) and 8.32 (6.64); final DMFT/S was 2.76 (2.84) and 3.01 (3.20). The missing (because of decay) second deciduous molars were most powerfully associated with caries increment during treatment, showing significant correlations to second premolars $(r=0.47, p=0.003)$, while fillings on second deciduous molars seem to influence the prevalence of fillings on permanent molars $(r=0.44, p=0.001)$. The increment at surface level was 2.01 (2.61) in the whole sample and 2.60 (3.81) in the high-risk group (4 boys, 6 girls). Compared to the low-risk group (10 girls), post-treatment caries experience was significantly higher in the high-risk group $(p=0.029)$. Boys were more at risk than girls $(p=0.005)$. Conclusions: Children with elevated caries experience in the early mixed dentition are exposed to higher caries risk during orthodontic treatment. Thus, in prevision of treatment, caries should already be assessed in the mixed dentition, so that an extended prophylaxis program can be initiated.
\end{abstract}

\section{Keywords}

Bitewing Radiography, Caries Increment, DMF Indices, Mixed Dentition, Orthodontic Appliances

How to cite this paper: Soumas, V., Kiliaridis, S. and Staudt, C.B. (2015) Is Caries in the Early Mixed Dentition Associated with Caries Development during Orthodontic Treatment? Journal of Biosciences and Medicines, 3, 25-32. 


\section{Introduction}

Although caries prevalence in children and young adults decreased dramatically in industrialized countries during the 1970s and 1980s [1] caries remains of concern in subgroups of the population. According to the American Academy of Pediatric Dentistry [2] patients wearing orthodontic appliances are considered at high risk for future caries development. In fact, demineralization around orthodontic brackets is known to be one of the undesired side effects of fixed orthodontic treatment [3]-[6]. Oral hygiene is made more difficult by fixed orthodontic appliances. A considerable mineral loss can take place without being observed clinically [6] because it is difficult to detect the lesions.

Therefore, patients with high demineralization and caries risk should be identified before starting orthodontic treatment, so that they can benefit from an extended prophylaxis program before and during the treatment. The first step in this approach is to determine valid predictors for individual demineralization and caries development during orthodontic treatment. Among the clinical variables in patients without orthodontic treatment, past caries experience in deciduous teeth is one of the most significant predictors of future caries development [7]. $\mathrm{Li}$ and Wang [8] found that children having caries in their primary teeth were nearly three times more likely to develop caries in their permanent teeth. The age span 5 - 10 years is especially crucial for the initiation and progression of caries on the mesial surface of the permanent first molar [9]. In the mixed dentition, the dmfs score, as a direct measure of caries experience, especially on deciduous molars, is a good predictor for caries development in the permanent dentition [7]. In the young permanent dentition, DFS score and occlusal morphology of first permanent molars become good predictors [7]. Before orthodontic treatment, adolescent and adult patients with high numbers of decayed and filled tooth surfaces (DFS) ran a higher risk of developing new cavities during treatment than those with a small number of DFS [10]. There is limited evidence concerning caries risk that young children in the early mixed dentition will encounter later during orthodontic treatment.

Thus, the objective of the present study is to investigate if caries experience in the early mixed dentition is associated with the development of caries in the permanent dentition during orthodontic treatment.

Our hypothesis is that children with higher caries experience in the mixed dentition are more at risk to develop caries in the permanent dentition during orthodontic treatment.

\section{Material and Methods}

The subjects were selected among the files of patients having finished their orthodontic treatment during the years 2003 to 2008 at our university clinic. Informed consent was obtained from the children's parents and ethical approval to use the data was obtained by the university hospital ethical research committee. Inclusion criteria to enter our study were radiographs and study casts to be available from the

a) initial pre-treatment documentation, taken in the early mixed dentition (deciduous molars not shed out physiologically; lower deciduous canines may have been shed out due to permanent canines starting eruption);

b) final documentation, taken in the permanent dentition after orthodontic treatment.

Forty-one patients, 29 girls and 12 boys met the inclusion criteria. Their mean age before starting orthodontic treatment was $9.4 \pm 1.1$ years and $14.9 \pm 1.6$ years at the final documentation after orthodontic treatment (Table 1). The orthodontic treatment was generally two-phased, consisting of a first phase with removable appliances followed by a second phase with fixed appliances. The total duration of treatment, from appliance placement until removal, including all pauses, was 4.5 years ( \pm 1.6 years). The final documentation was undertaken 6.2 months ( \pm 5.4 months) after the removal of the appliances. Patients with syndromes were excluded.

\section{Table 1. Sample description.}

\begin{tabular}{ccccc}
\hline & \multicolumn{4}{c}{ Years (mean, SD) } \\
\cline { 2 - 5 } Patients & Number & Age pre-treatment & Age post-treatment & Treatment duration \\
\cline { 2 - 5 } Girls & 29 & $9.2(0.93)$ & $14.7(1.43)$ & $4.6(1.65)$ \\
Boys & 12 & $9.6(1.30)$ & $15.3(2.00)$ & $4.4(1.54)$ \\
All & 41 & $9.4(1.08)$ & $14.9(1.61)$ & $4.5(1.60)$ \\
\hline
\end{tabular}




\subsection{Data Collection}

The index used for measuring caries experience was the $\mathrm{dmft} / \mathrm{s}$ on deciduous and the DMFT/S on permanent teeth, assessing the number of decayed, missing and filled teeth/tooth surfaces. On the pre-treatment radiographs taken in the mixed dentition, the deciduous molars $(04,05)$ and the permanent first molars $(6)$ were assessed. On the post treatment radiographs taken in the permanent dentition, the bicuspids $(4,5)$ and permanent molars $(6,7)$ were assessed. The condition of the occlusal, mesial and distal surface was recorded for each tooth.

\subsection{Present and Missing Teeth/Surfaces (m, M)}

The presence of erupted and unerupted teeth was assessed on the orthopantomograms and verified on study casts. Patient records were examined in order to discern missing teeth due to caries from missing teeth due to trauma, extraction for orthodontic reasons, undermining resorption or agenesis.

\subsection{Decayed (d, D) and Filled (f, F) Teeth/Surfaces}

The presence of caries (d, D) and fillings (f, F) was assessed on bitewing radiographs (Figure 1) on a light box in a dark room. Calibration and training sessions took place before the initial caries examination by one author (VS). All radiolucent discontinuation of enamel on deciduous and permanent teeth visible on X-rays was noted as decay. For occlusal surfaces, dentinal and enamel/dentin border caries were included. If a tooth surface had both a caries lesion and a filling, it was considered as decayed only. Thus, when a first permanent molar was filled before orthodontic treatment and developed caries during treatment, post-treatment caries is noted. Fissure sealed teeth were classified as sound teeth.

\subsection{High-Risk and Low-Risk Group}

A high-risk and a low-risk group were created by sorting the patients according to initial dmfs + DMFS score before treatment. Approximately 25\% (10/41) (4 boys, 6 girls) of the patients with the highest number of surfaces affected were included into the high-risk group. The patients with the lowest caries experience before treatment (25\%, 10/41; 10 girls) were included into the low-risk group.

\subsection{Statistical Analysis}

The dmft/s and DMFT/S indices (the sum of decayed, missed and filled teeth/surfaces in temporary and permanent dentition) were calculated and descriptive analysis as well as correlation (Kendall tau) and multiple linear

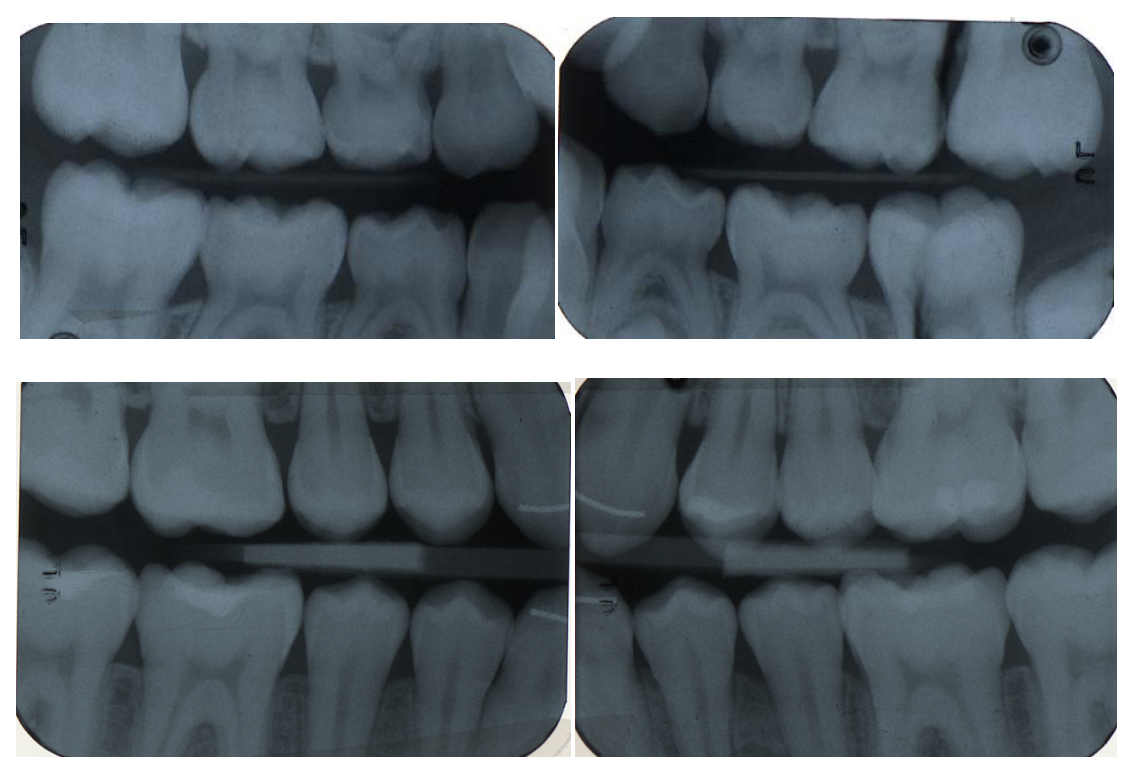

Figure 1. Bitewing radiographs pretreatment and posttreatment. 
regression analyses were performed (SPSS, version 15.0, Inc. Chicago IL). Mann-Whitney U-test was used to determine any significant difference between the groups. The level of statistical significance was set at 0.05 . Two months after the initial evaluation, the radiographs of 20 randomly selected patients were re-assessed by one author (VS). Unweighted kappa statistics was performed to measure the reproducibility.

\section{Results}

The agreement between the first and the repeated measurement was excellent when evaluating bitewing radiographs for decay $(\kappa=0.95)$ and fillings $(\kappa=1.00)$.

At the pre-treatment documentation all first permanent molars and almost all permanent incisors (95.1\%) had erupted. By the end of the treatment all permanent teeth had erupted except for $4.9 \%$ of the second molars and all third molars.

There were more girls (29) than boys (12) in our sample (Table 1$)$ and their mean age $(\mathrm{p}=0.177)$ and treatment time $(\mathrm{p}=0.694)$ were not statistically different. Boys showed statistically significant higher DMFT/S scores after orthodontic treatment (5.00, SD 2.67/5.58, SD 2.85) than girls (1.83, SD 1.48/1.97, SD 1.62) $(\mathrm{p}=$ $0.002 / 0.001)$ and over three times more DMFS increment (3.92, SD 2.42) than girls (1.25, SD 1.18) ( $\mathrm{p}=0.005)$.

\subsection{Caries Experience}

The percentage of caries free patients $(\mathrm{dmfs} / \mathrm{dmfS}=0)$ was $9.8 \%(\mathrm{n}=4)$ at the initial documentation and $22.0 \%$ $(n=9)$ at the end of orthodontic therapy.

At tooth level, pre-treatment caries experience in the mixed dentition was 5.15 (SD 3.60) and post-treatment caries experience in the permanent dentition was 2.76 (SD 2.84). At surface level (Table 2(a) and Table 2(b)), the scores were 8.32 (SD 6.64) and 3.01 (SD 3.20), respectively. At both instances, the decayed and filled component accounted similarly to the score, whereas missing teeth because of caries were found only pre-treatment (0.95, SD 2.37). Post-treatment caries experience (Table 3) was significantly higher in the high-risk than in the low-risk group $(\mathrm{p}=0.029)$.

\subsection{Before Treatment}

Before treatment, in the mixed dentition, $54.0 \%$ of the deciduous molars and $27.4 \%$ of the first permanent molars were affected by caries. Over six times more surfaces were affected on the deciduous molars than in permanent first molars (Table 2(a)). The proximal surfaces of the deciduous molars were twice as often decayed as the occlusal surfaces, whereas the occlusal surfaces of the first permanent molars were nearly five times as often decayed or filled as the proximal surfaces. Decayed deciduous molars were often left untreated, whereas three quarters of the decayed permanent first molars had fillings.

\subsection{After Treatment}

After treatment, in the permanent dentition, $41.4 \%$ of the first permanent molars were affected by caries, thus $14 \%$ more than before the orthodontic treatment. When recording the caries experience after treatment, about two out of the three surfaces affected were on the first molars and the remaining one was on the second premolars or molars (Table 2(b)). On the premolars, predilection sites for caries were rather proximally, on the molars occlusally. Again, only the first permanent molars were treated in most cases.

\subsection{Increment}

The overall increment of caries experience during treatment was 2.01 (2.61). On average, one new surface was affected on a first molar and one new surface either on a second premolar or second molar (Table 4). In the high-risk group, the DMFS increment was 2.60 (3.81) (Table 3), with a maximum score of 13 in one patient and no increment (score 0 ) in two patients. In the low-risk group, the mean increment was also important (2.00 (2.36)) and very close to the mean for all patients, with a maximum score of 6 in one patient, and no increment in three patients. The high-risk and the low-risk groups did not statistically differ as to the increment on caries experience $(p=0.809)$; neither did the high-risk group differ from the entire sample $(p=0.730)$. 
Table 2. (a) and (b) Caries experience pre-treatment (II A) and post-treatment (II B) at surface level (dmfs/DMFS) by tooth type (04, 05, 6 and 4, 5, 6, 7, respectively) and surface type (occlusal, proximal).

(a)

\begin{tabular}{|c|c|c|c|c|}
\hline & \multicolumn{4}{|c|}{ Caries experience pre-treatment: Number of affected tooth surfaces } \\
\hline & Decayed & Missing & Filled & dmfs/DMFS \\
\hline Tooth type & Mean (SD) & Mean (SD) & Mean (SD) & Mean (SD) \\
\hline 04 & $1.83(2.05)$ & $0.51(1.49)$ & $1.32(2.21)$ & $3.66(3.14)$ \\
\hline Occlusal & $0.61(0.86)$ & . & $0.66(1.09)$ & $1.27(1.28)$ \\
\hline Proximal & $1.22(1.47)$ & . & $0.66(1.17)$ & $1.88(1.71)$ \\
\hline 05 & $1.81(1.96)$ & $0.44(1.27)$ & $1.41(2.33)$ & $3.66(3.34)$ \\
\hline Occlusal & $0.61(1.02)$ & . & $0.78(1.24)$ & 1.39 (1.50) \\
\hline Proximal & $1.20(1.21)$ & . & $0.63(1.26)$ & $1.83(1.61)$ \\
\hline 6 & $0.27(0.55)$ & $0.0(0.0)$ & $0.73(1.30)$ & $1.00(1.43)$ \\
\hline Occlusal & $0.17(0.50)$ & . & $0.66(1.24)$ & $0.83(1.24)$ \\
\hline Proximal & $0.10(0.30)$ & . & $0.07(0.35)$ & $0.17(0.54)$ \\
\hline $\operatorname{Sum}(04,05,6)$ & $3.91(3.20)$ & $0.95(2.37)$ & $3.46(4.70)$ & $8.32(6.64)$ \\
\hline
\end{tabular}

(b)

\begin{tabular}{|c|c|c|c|c|}
\hline & \multicolumn{4}{|c|}{ Caries experience post treatment: Number of affected tooth surfaces } \\
\hline & Decayed & Missing & Filled & DMFS \\
\hline Tooth type & Mean (SD) & Mean (SD) & Mean (SD) & Mean (SD) \\
\hline 4 & $0.04(0.22)$ & $0.0(0.0)$ & $0.0(0.0)$ & $0.04(0.22)$ \\
\hline Occlusal & $0.02(0.16)$ & . & $0.0(0.0)$ & $0.02(0.16)$ \\
\hline Proximal & $0.02(0.16)$ & . & $0.0(0.0)$ & $0.02(0.16)$ \\
\hline 5 & $0.44(1.03)$ & $0.0(0.0)$ & $0.04(0.31)$ & $0.48(1.05)$ \\
\hline Occlusal & $0.12(0.40)$ & . & $0.02(0.16)$ & $0.15(0.42)$ \\
\hline Proximal & $0.32(0.79)$ & . & $0.02(0.16)$ & $0.34(0.79)$ \\
\hline 6 & $0.64(0.83)$ & $0.0(0.0)$ & $1.29(1.74)$ & $1.93(1.79)$ \\
\hline Occlusal & $0.54(0.78)$ & . & $1.07(1.44)$ & $1.61(1.46)$ \\
\hline Proximal & $0.10(0.37)$ & . & $0.22(0.69)$ & $0.32(0.88)$ \\
\hline 7 & $0.34(0.62)$ & $0.0(0.0)$ & $0.22(0.76)$ & $0.56(1.03)$ \\
\hline Occlusal & $0.27(0.50)$ & . & $0.22(0.76)$ & $0.49(0.87)$ \\
\hline Proximal & $0.07(0.35)$ & . & $0.0(0.0)$ & $0.07(0.35)$ \\
\hline $\operatorname{Sum}(4,5,6,7)$ & $1.46(1.80)$ & $0.00(0.0)$ & $1.55(2.13)$ & 3.01 (3.20) \\
\hline
\end{tabular}


Table 3. Caries experience pre- and post-treatment as well as caries increment at surface level (dmfs/DMFS) for the high-risk and de low-risk group, compared to the whole sample.

\begin{tabular}{cccccc}
\hline & & & \multicolumn{3}{c}{$\mathrm{dmfs} /$ DMFS (mean, SD) } \\
\hline Group & Number & (Female, male) & Pre-treatment & Post-treatment & Increment \\
\hline High risk & 10 & $(4 \mathrm{~m}, 6 \mathrm{f})$ & $18.10(4.72)$ & $5.00(4.40)$ & $2.60(3.81)$ \\
Low risk & 10 & $(10 \mathrm{f}, 0 \mathrm{~m})$ & $1.20(1.23)$ & $2.00(2.40)$ & $2.00(2.36)$ \\
All patients & 41 & $(29 \mathrm{f}, 12 \mathrm{~m})$ & $8.32(6.64)$ & $3.01(3.20)$ & $2.01(2.61)$ \\
\hline
\end{tabular}

Table 4. Increment in caries experience at surface level (DMFS) by tooth type $(4,5,6,7)$ and surface type (occlusal, proximal).

\begin{tabular}{|c|c|c|c|c|}
\hline & \multicolumn{4}{|c|}{ Caries increment: Number of new affected tooth surfaces } \\
\hline & Decayed & Missing & Filled & DMFS \\
\hline Tooth type & Mean (SD) & Mean (SD) & Mean (SD) & Mean (SD) \\
\hline 4 & $0.04(0.22)$ & $0.0(0.0)$ & $0.0(0.0)$ & $0.04(0.16)$ \\
\hline Occlusal & $0.02(0.16)$ & . & $0.0(0.0)$ & $0.02(0.16)$ \\
\hline Proximal & $0.02(0.16)$ & . & $0.0(0.0)$ & $0.02(0.16)$ \\
\hline 5 & $0.44(1.03)$ & $0.0(0.0)$ & $0.05(0.31)$ & $0.48(1.05)$ \\
\hline Occlusal & $0.12(0.40)$ & . & $0.02(0.16)$ & $0.15(0.42)$ \\
\hline Proximal & $0.32(0.79)$ & . & $0.02(0.16)$ & $0.34(0.79)$ \\
\hline 6 & $0.37(0.94)$ & $0.0(0.0)$ & $0.56(0.74)$ & $0.93(1.17)$ \\
\hline Occlusal & $0.37(0.94)$ & . & $0.41(0.92)$ & 0.78 (1.01) \\
\hline Proximal & $0.00(0.00)$ & . & $0.15(0.42)$ & $0.15(0.48)$ \\
\hline 7 & $0.34(0.62)$ & $0.0(0.0)$ & $0.22(0.76)$ & $0.56(1.03)$ \\
\hline Occlusal & $0.27(0.50)$ & . & $0.22(0.76)$ & $0.49(0.87)$ \\
\hline Proximal & $0.07(0.35)$ & . & $0.0(0.0)$ & $0.07(0.35)$ \\
\hline $\operatorname{Sum}(4,5,6,7)$ & $1.19(1.99)$ & $0.0(0.0)$ & $0.82(1.14)$ & $2.01(2.61)$ \\
\hline
\end{tabular}

\subsection{Correlations}

No statistically significant correlations were found between overall caries experience in the mixed dentition before orthodontic treatment and increment in caries experience in the permanent dentition after orthodontic treatment. When focusing on the dmft score of deciduous molars, correlations were found with the score of the second premolars and first molars $(r=0.30, p=0.022$ and $r=0.28, p=0.024$, respectively). Second deciduous molars score taken apart was correlated with the score at first permanent molars after treatment $(\mathrm{r}=0.38, \mathrm{p}=$ 0.003). At surface level, correlations were obtained between the first deciduous molars' proximal fillings and the proximal fillings experience on the first permanent molars after treatment $(r=0.48, p=0.001)$. Decayed permanent first molars before treatment (proximal lesions) were significantly correlated to caries increment during orthodontic treatment on the second molars (also proximal lesions) $(r=0.31, \mathrm{p}=0.045)$. When deciduous molars were missing because of caries, especially the second ones, their score was significantly correlated to caries 
experience or fillings on the second premolars $(r=0.47, p=0.003)$. Pre-treatment fillings on the second deciduous molars seem to influence the prevalence of fillings on first molars after treatment $(r=0.44, p=0.001)$.

\subsection{Multiple Regression Analysis}

By performing a multiple regression analysis, a significant model emerged (adjusted $\mathrm{R}^{2}=0.179, \mathrm{~F}_{3,37}=3.907, \mathrm{p}$ $=0.016)$ where the variable sex $(B=0.492, p=0.004)$ could explain $17.9 \%$ of the variability in overall caries increment. This model could not be improved significantly by the combination with further variables (age, treatment duration, caries experience).

\section{Discussion}

The present longitudinal cohort study revealed a significant positive association between caries experience in the early mixed and in the permanent dentition during orthodontic treatment. Due to the retrospective character of our study, caries detection was purely based on bitewing radiographs, where we achieved very good reproducibility. Although this is the standard method for approximal caries detection [11], underestimation of the prevalence of occlusal and bucco/lingual caries requiring clinical examination cannot be excluded.

In the present study, pre-treatment caries experience in the mixed dentition was relatively high (5.15 at tooth level, 8.32 at surface level), when compared to Scandinavian children (2.43 and 3.53 [12] and to similar aged (10 years) Swiss children from Zurich in 2005 (0.45 and 0.55) [13]. We are aware that the population asking for treatment in our Dental School with income-related fee reduction is not representative for the canton of Geneva or even for the Geneva's population in terms of socio-economic background. We considered that this difference was favoring our study since relatively high pre-treatment caries activity could have increased the probability of revealing correlations to the post-treatment situation.

In contrast to the associations found in the literature for various dentition stages (without orthodontic treatment: 5 vs. 10 year olds [14] or 3.5 vs. 11.7 year olds [8]; permanent dentition before and after orthodontic treatment [10]), in the present study no significant correlation was found between overall caries experience in the mixed and in the permanent dentition after orthodontic treatment. The deciduous molars, with an over six times higher caries experience than the permanent first molars, were the most powerful variables at baseline. They are known to be good predictors for caries development in the permanent dentition [7] [8] [14]. In the present study, their caries experience was significantly correlated to the premolars and first molars and the more often they were missing due to caries, the more often the second premolars were affected. These correlations confirmed our hypothesis that children with higher caries experience in the mixed dentition are more at risk to develop caries in the permanent dentition during orthodontic treatment.

During orthodontic treatment, when oral hygiene is more challenging, an increment in caries experience of 2.01 was observed in the entire sample. In the high-risk group, the increment was more elevated (2.60), but statistical significance was not reached neither in respect to the entire sample (2.01) nor in respect to the low risk group (2.00). However, post-treatment caries experience was significantly higher in the high-risk (5.00) than in the low-risk group (2.00). In accordance with the study of Hansel Petersson [15], where 56.2\% of the children (11 to 12 year old) developing new caries were boys, a higher percentage of boys $(4 / 12=33 \%)$ than girls $(6 / 29$ $=2.7 \%$ ) was attributed to the high risk group in our study, whereas no boys were represented in the low risk group. Accordingly, the variability in overall caries increment was explained by sex, to nearly $18 \%$.

\section{Conclusion}

Children with elevated caries experience in the early mixed dentition, especially on deciduous second molars and permanent first molars, are exposed to a higher risk to develop caries in the permanent dentition during orthodontic treatment (mainly on the second premolars and first molars). Caries increment was higher in boys than in girls. Thus, in the light of the findings in this retrospective study and in prevision of orthodontic treatment, caries activity should be assessed in the mixed dentition, in order to already involve the patient in an extended prophylaxis program.

\section{References}

[1] Marthaler, T.M. (2004) Changes in Dental Caries 1953-2003. Caries Research, 38, 173-181. 
http://dx.doi.org/10.1159/000077752

[2] The American Academy of Pediatric Dentistry (AAPD) (2002) Policy Statement on the Use of a Caries-Risk Assessment Tool (CAT). Council on Clinical Affairs.

[3] Gorelick, L., Geiger, A.M. and Gwinnet, A.J. (1982) Incidence of White Spot Formation after Bonding and Banding. American Journal of Orthodontics, 81, 93-98. http://dx.doi.org/10.1016/0002-9416(82)90032-X

[4] Årtun, J. and Brobakken, B.O. (1986) Prevalence of Carious White Spots after Orthodontic Treatment with Multibonded Appliances. European Journal of Orthodontics, 8, 229-234. http://dx.doi.org/10.1093/ejo/8.4.229

[5] Øgaard, B. (1989) Prevalence of White Spot Lesions in 19-Year-Olds: A Study on Untreated and Orthodontically Treated Persons 5 Years after Treatment. American Journal of Orthodontics and Dentofacial Orthopedics, 96, 423427. http://dx.doi.org/10.1016/0889-5406(89)90327-2

[6] O’Reilly, M.M. and Featherstone, J.D.B. (1987) Demineralization and Remineralization around Orthodontic Appliances: An in Vivo Study. American Journal of Orthodontics and Dentofacial Orthopedics, 92, 33-40. http://dx.doi.org/10.1016/0889-5406(87)90293-9

[7] Powell, L.V. (1998) Caries Prediction: A Review of the Literature. Community Dentistry and Oral Epidemiology, 26, 361-371. http://dx.doi.org/10.1111/j.1600-0528.1998.tb01974.X

[8] Li, Y. and Wang, W. (2002) Predicting Caries in Permanent Teeth from Caries in Primary Teeth: An Eight-Year Cohort Study. Journal of Dental Research, 81, 561-566. http://dx.doi.org/10.1177/154405910208100812

[9] Mejàre, I. and Stenlund, H. (2000) Caries Rates for the Mesial Surface of the First Permanent Molar and the Distal Surface of the Second Primary Molar from 6 to 12 Years of Age in Sweden. Caries Research, 34, 454-461. http://dx.doi.org/10.1159/000016623

[10] Al Mulla, A.H., Al Kharsa, S., Kjellberg, H., et al. (2009) Caries Risk Profiles in Orthodontic Patients at Follow-Up Using Cariogram. Angle Orthodontics, 79, 323-330. http://dx.doi.org/10.2319/012708-47.1

[11] Kidd, E.A. and Pitts, N.B. (1990) A Reappraisal of the Value of the Bitewing Radiograph in the Diagnosis of Posterior Approximal Caries. British Dental Journal, 169, 195-200. http://dx.doi.org/10.1038/sj.bdj.4807325

[12] Skeie, M.S., Raadal, M., Strand, G.V., et al. (2006) The Relationship between Caries in the Primary Dentition at 5 Years of Age and Permanent Dentition at 10 Years of Age-A Longitudinal Study. International Journal of Paediatric Dentistry, 16, 152-160. http://dx.doi.org/10.1111/j.1365-263X.2006.00720.x

[13] Steiner, M., Menghini, G., Marthaler, T.M., et al. (2010) Changes in Dental Caries in Zurich School-Children over a Period of 45 Years. Schweiz Monatsschr Zahnmed, 120, 1084-1104.

[14] Skeie, M.S., Raadal, M., Strand, G.V., et al. (2004) Caries in the Primary Teeth at 5 and 10 Years of Age: A Longitudinal Study. European Journal of Paediatric Dentistry, 4, 194-202.

[15] Hansel Petersson, G., Twetman, S. and Bratthall, D. (2002) Evaluation of a Computer Program for Caries Risk Assessment in Schoolchildren. Caries Research, 36, 327-340. http://dx.doi.org/10.1159/000065963 